\title{
PARIETAL CORTEX AND COGNITION
}

\section{Preface}

This special issue was organized to highlight the importance of the parietal cortex in mediating cognitive processes associated with perceptual memory, attention, and egocentric and allocentric spatial long-term memory representations in rats and monkeys. The first three articles emphasize the importance of a selective attention role for the posterior parietal cortex. The first article, by Corwin and Reep, entitled "Rodent Posterior Parietal Cortex as a Component of a Cortical Network Mediating Directed Spatial Attention," provides for an anatomical definition of the posterior parietal cortex in the rat and its role in directed spatial attention. The second article, by Crowne and Mah, entitled "A Comparison of Hemispatial Neglect From Posterior Parietal and Periarcuate Lesions in the Monkey," provides further evidence for a role of the posterior parietal cortex in monkeys in subserving spatial attention based on the presence of hemispatial neglect. The third article, by Steinmetz, entitled "Contributions of Posterior Parietal Cortex to Cognitive Functions in Primates," summarizes electrophysiological evidence based on neural activity of parietal cortex cells in monkeys in mediating a variety of functions, including selective attention.

The next two articles emphasize the importance of the binding properties of the posterior parietal cortex as an index of attention. Chen and Nakamura, in an article entitled "Head-Centered Representation and Spatial Memory in Rat Posterior Parietal Cortex," suggest on the basis of single-unit activity data that the parietal cortex integrates vestibular input, motion, and direction to represent head- or body-centered space. Long, Mellem, and Kesner, in an article entitled "The Effects of Parietal Cortex Lesions on an Object/Spatial Location Paired-Associate Task in Rats," demonstrate that lesions of the posterior parietal cortex do not disrupt visual object or spatial location discrimination learning, but do disrupt learning when both visual object and spatial location are of importance to solve the task, suggesting that the posterior parietal cortex might bind specific features together perhaps through the use of attentional processes.

In the next article, Kolb and Cioe show that there is little recovery of function following neonatal injury of the posterior parietal cortex in comparison with other neural regions, suggesting reduced plasticity of the posterior parietal cortex in response to injury.

In the last three articles, there is an emphasis on the role of the posterior parietal cortex in representing spatial information. In the article by McDaniel, Williams, Attaway, and Compton, entitled "Turn-Signal Utilization by Rats With Either Unilateral or Bilateral Posterior Parietal Cortex Injuries," it is suggested on the basis of several studies that the posterior parietal cortex is primarily involved in the integration of kinesthetic information to sustain egocentric spatial processes. In the article by Save, Poucet, Foreman, and Thinus-Blanc, entitled "The Contribution of the Associative Parietal Cortex and Hippocampus to Spatial Processing in Rodents," there is a summary of the rodent literature on the role of the posterior parietal cortex in processing spatial information. They suggest that the critical role of the posterior parietal cortex is to associate visual and spatial information with internal movement-related information to translate egocentric frames of reference into allocentric spatial representations. In the last article, entitled "Parietal Cortex and a Spatial Cognitive Map," Kesner and Long suggest that on the basis of perceptual memory processes, attentional processes, and egocentric and allocentric spatial processes, the posterior parietal cortex serves as a long-term store for a spatial cognitive map. It is to be hoped that the publication of this special issue will engender an increased interest in the role of the posterior parietal cortex in mediating cognitive functions. 